\title{
El balance hídrico en cuerpos de agua cársticos de la Península de Yucatan
}

DOI: $10.22403 /$ UROOMX/TYP03/12

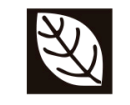

Resumen

Adrián Cervantes Martínez*

Debido a la naturaleza calcárea de la Península de Yucatán (PY) sus suelos se caracterizan por ser porosos y altamente permeables; razón por la cual no se forman corrientes superficiales de importancia y el flujo de agua es mayoritariamente subterráneo. Para el mejor uso y manejo del recurso agua es necesario conocer la recarga y descarga a través de la precipitación, evapotranspiración y escorrentía. La obtención de estas variables se facilita en cuencas con baja permeabilidad del suelo, debido a que dominan corrientes superficiales; sin embargo, en regiones donde los flujos son principalmente subterráneos (como en los sistemas cársticos), los cálculos hidrológicos se pueden dificultar. En este trabajo se presenta un esbozo general del estado del conocimiento del balance hídrico $(\mathrm{Bh})$ generado para la Py, el cual es escaso y contradictorio. Se resaltan, además, las experiencias geohidrológicas de otras regiones cársticas del mundo, así como las técnicas para el mejor cálculo del Bh en estos ambientes. Se mencionan y discuten también, los factores que limitan el conocimiento del $\mathrm{Bh}$ en los cuerpos de agua cársticos de la PY.

Palabras | Balance hídrico, cárstico, cenote, flujo subterráneo, geohidrología. CLAVE

* Universidad de Quintana Roo, Cuerpo Académico de Turismo / adcervantes@uqroo.mx 


\section{Introducción}

El balance hídrico es un término que se refiere a la relación entre la ganancia y pérdida de agua en forma de precipitación, evapotranspiración y escorrentía (flujo superficial y subterráneo) que ocurre en una región en particular (Murat, 1998: 405). La obtención de estas variables se facilita en cuencas con baja permeabilidad del suelo, debido a que dominan corrientes superficiales. Sin embargo, en regiones donde dominan suelos con alta porosidad y por ende, flujos mayoritariamente subterráneos (como en los sistemas cársticos), los cálculos hidrológicos se pueden dificultar (CNA, 2002; Medina y Herrera, 2003: 457).

A nivel local y regional, en ambientes cársticos y no cársticos, se pueden encontrar diferencias en la capacidad de almacenaje, cantidad de agua drenada, recarga y descarga de los tributarios, composición química del agua, etc., producidas por las variaciones en la precipitación, clima y orografía de cada lugar (Perrin et al., 2003: 677).

La Península de Yucatán (PY) es una región compuesta por carbonatos del Terciario (Lesser y Weidie, 1988) que permiten la formación de una gran cantidad de sistemas superficiales denominados cenotes, y subterráneos como las cavernas. La región se caracteriza por una alta porosidad del suelo que limita la formación de corrientes superficiales y el flujo del agua, principalmente subterráneo (Alcocer y Escobar, 1996: 62; Herrera y Comín, 2000: 215; Schmitter et al., 2002: 215 ). Así, el balance hídrico probablemente es diferente entre localidades de la región, incluso variable entre sistemas acuáticos (por ejemplo, en sistemas lóticos y lénticos), debido a:

a) Diferencias en precipitación: mayor en la zona sur (I500 mm) y menor en la norte $(500 \mathrm{~mm})$ (Herrera y Comín, 2000) (Schmitter et al., 2002).

b) Diferencias en el flujo y velocidad de escorrentía: sólo en el noroeste de la PY se ha identificado la presencia de algunas cuencas que se consideran lénticas; cuando el gradiente hidráulico es bajo, el agua recorre de 7 a $10 \mathrm{~mm} / \mathrm{d}$, porque fluye a través de grietas pequeñas. Sin embargo, en la misma zona existen cenotes conectados a un sistema de flujo subterráneo horizontal, donde la masa de agua dulce se desplaza unidireccionalmente, impulsada por la gravedad a través de 
canales amplios $(>0.5 \mathrm{~m})$ donde la velocidad de flujo es alta $(3 \mathrm{~cm} / \mathrm{s})$, constituyendo sistemas lóticos (Schmitter et al., 2002: 2 I 8). Por otro lado, en la región centro y sur de la PY sólo se han hecho conjeturas sobre los tipos y velocidad de flujo del agua subterránea.

c) Diferencias en la intrusión de agua marina hacia el continente: en el nor-noroeste de la PY se ha generalizado que una capa delgada de agua dulce flota sobre una de agua salina más densa y que la intromisión de esta última alcanza hasta $110 \mathrm{~km}$ dentro de la PY. Esto explica la presencia de haloclinas en sistemas muy cercanos a la costa (Alcocer et al., 1998: 298); sin embargo, en sistemas del centro-sur de Quintana Roo, pero a una distancia de $25-30 \mathrm{~km}$ del mar, no se han registrado haloclinas (Cervantes et al., 2002: I75), evidenciando que la intromisión de agua marina en la PY es diferente en la región.

Lo anterior, por un lado muestra que existe falta de información acerca de patrones de flujo subterráneo y, por otro, dificulta la generación de información básica de descarga de agua, por localidades o por cuencas, impidiendo el cálculo preciso del balance hídrico a nivel local y regional en la PY.

Condiciones muy diferentes se encuentran en otras regiones cársticas del mundo, como Turquía, Suiza y Florida, donde ya se han identificado los promedios, máximos y mínimos de descarga y recarga de agua subterránea de acuerdo con la estacionalidad, volumen de agua que aportan los tributarios al acuífero, incluso cambios en el flujo debido a otros sistemas cársticos de la zona o fallas tectónicas (Murat, 1999:4 I 2; Martin y Screaton, 200 I; Perrin et al., 2003: 673). En la PY, por el contrario, este tipo de conocimiento es incipiente, sólo en la región Noroeste se ha establecido que el flujo dominante es de sureste a noroeste (Steinich y Marín, 1997: 328), pero dos terceras partes de la PY se consideran inexploradas (Schmitter et al., 2002: 2I7); este problema se discutirá con mayor profundidad más adelante, pero es indudable la necesidad de evaluar los patrones de circulación subterránea, evapotranspiración, regímenes de descarga, así como las características físicas y químicas del agua de estas regiones cársticas.

A continuación, y con base en lo descrito y conocido para otras partes del mundo, se exponen algunas herramientas que podrían ser útiles para precisar el balance hídrico en los sistemas cársticos. 
Balance hídrico: el presupuesto de agua de una cuenca, considera tres parámetros: precipitación, evapotranspiración y escorrentía; por razones obvias, este último es más fácil de evaluar superficialmente; pero en aguas subterráneas es más complicado, debido a que en la mayoría de los casos los límites entre agua subterránea y superficial no son claros. El balance hídrico del agua, en los sistemas cársticos se obtiene a partir de la siguiente ecuación:

$\Delta \mathrm{Q}=\mathrm{P}-\mathrm{ET}-\mathrm{Sr}$, donde

$\mathrm{P}=$ precipitación

$\mathrm{ET}=$ Evapotranspiración

$\mathrm{Sr}=$ es la recarga o descarga de agua

$\Delta \mathrm{Q}=$ balance hídrico

Para determinar $\mathrm{Sr}$ es necesario calcular los regímenes de descarga y tiempo de residencia del agua, los cuales se definen a continuación:

Régimen de descarga: también conocido como alimentación a los acuíferos cársticos. Es una variable que está en función del volumen de agua almacenada $\left(\mathrm{V}_{\mathrm{s}}\right)$ (Perrin et al., 2003: 678) y se puede obtener a partir de la siguiente ecuación:

$\mathrm{Qt}=\mathrm{Q}_{0}{ }^{*} \mathrm{e}_{-}{ }^{\alpha(\mathrm{t}-\mathrm{to})}$, donde

$\mathrm{Qt}=$ descarga en $\mathrm{m}^{3} \mathrm{~s}^{-1}$ en el tiempo $\mathrm{t}$

$\mathrm{Qo}=$ es la descarga previa de agua, al tiempo cero

to= el tiempo entre Qt y Qo

$\mathrm{e}=$ la base del logaritmo natural

y $\alpha=$ al coeficiente de recesión del agua (descarga)

Los valores de $\alpha$ derivan de las características hidrológicas del acuífero y son de gran importancia en los sistemas cársticos debido a la alta porosidad del suelo. Este término representa la capacidad del acuífero para liberar agua, por ejemplo, valores bajos de $\alpha$ indican una tasa de drenado baja y gran capacidad de almacenaje (condición que probablemente se presente en cenotes lénticos); por el contrario, valores altos de $\alpha$, indican una tasa rápida de drenado y una baja capacidad de almacenado (en sistemas lóticos) (Martin y Screaton, 200 I; Perrin et al., 2003: 680).

Por lo tanto, los valores de $\alpha$ son dependientes del tipo de roca del subsuelo. Se ha observado que sistemas cársticos de Turquía tienen una gran capacidad de almacenaje y tasas de drenado bajas (Murat, 1998:407). En sistemas 
cársticos de la Florida, el subsuelo poroso funciona como sistema de almacenaje, mientras que el flujo y transporte del agua subterránea se presenta en suelos con conductos o galerías. Por lo tanto, el flujo subterráneo está relacionado con la agresividad de la disolución de la roca calcárea y la subsiguiente formación de conductos o galerías (Armengol, 1997:10; Maramathas et al., 2003: 610). Así, la dinámica del agua subterránea será controlada principalmente por dos factores hidrogeológicos: la carstificación y las fallas tectónicas.

Evapotranspiración: puede medirse por el método de cloro ( $\mathrm{Cl}-$ ) (Appelo y Potsma, citado en Murat, 1998: 407). Este método se basa en la concentración de cloro que presentan el agua subterránea, la precipitación y acuíferos expuestos (donde cada uno tiene diferentes concentraciones de este ión). Es importante mencionar que estos valores deben ser comparados con estaciones climatológicas cercanas a los flujos de agua estudiados, ya que puede haber diferencias si éstos se comparan con valores de regiones más distantes. La ecuación es la siguiente:

$\mathrm{ET}=\mathrm{I}-(\mathrm{Cl}-\mathrm{p} / \mathrm{Cl}-\mathrm{s}+\mathrm{e})$, donde:

$\mathrm{ET}=$ evapotranspiración (\%)

$\mathrm{Cl}_{\mathrm{p}}=$ concentración de $\mathrm{Cl}$ de la precipitación (mmol L-1).

$\mathrm{Cl}^{\mathrm{P}}{ }_{\mathrm{s}+\mathrm{e}}=$ concentración de $\mathrm{Cl}$ del agua subterránea $\mathrm{y}$ acuíferos expuestos.

Evaluar el balance hídrico en los sistemas cársticos de la PY puede ser complicado, debido a que los regímenes de descarga y el tiempo de residencia del agua no pueden entenderse bien hasta que se conozcan a fondo los patrones de circulación subterránea a nivel local y regional. La única región que ha sido explorada en este sentido es la noroeste (particularmente el anillo de cenotes de Yucatán), donde se ha identificado un flujo sureste-noroeste; pero además se han detectado zonas altamente variables, donde la dirección del flujo, incluso, puede invertirse de una estación climática a otra (Steinich et al., 1996: 157; Steinich y Marín, 1997: 322).

La situación se complica más cuando encontramos cuerpos de agua muy cercanos entre sí que presentan diferencias en sus características físicas y químicas (Armengol y Miracle, 1997: I5; Cervantes et al., 2002: I75; Perrin et al., 2003: 676). La complicada geohidrología de las tierras cársticas produce que muchos de sus descriptores ambientales tengan gran variabilidad (Lavat et al., 200I: 607). En este sentido es necesario evaluar las variables físicas y 
químicas de las aguas subterráneas en tributarios y sistemas adyacentes, incluso dentro de los mismos sistemas (lagunas con cenotes dentro) con el objetivo de conocer qué variables controlan la diferenciación espacial, o qué variables pueden usarse como elementos traza para determinar el flujo subterráneo, de los cuales se hablará más adelante.

Hasta hoy, la única fuente de información para conocer la dirección del flujo subterráneo en la $P Y$ ha sido la diferencia en altitud con respecto al área de captura (CNA, 2002; Suárez y Rivera, 2000: I 55). Los flujos se han construido bajo el supuesto de que la variabilidad temporal sobre una escala estacional, permitirá tener una idea del tiempo de residencia en el acuífero, incluso un evento de inundación (llenado) puede indicar la ocurrencia de una rápida infiltración. Con esta información se ha determinado el siguiente balance hídrico para los cenotes de la PY (CNA, 2002; Herrera y Comín, 2000: 679; Schmitter et al., 2002: 217):

El ciclo del agua en la región muestra una circulación vertical dominante entre la atmósfera y el subsuelo, debido a que el suelo es muy delgado y altamente permeable. Por lo tanto, el agua de lluvia se infiltra rápidamente y la evaporación es alta. La precipitación como principal fuente alimentadora es diferente entre regiones, ya que puede variar entre $500-1500 \mathrm{~mm}$ en la región sur y norte, respectivamente, lo cual representa 172 I58 5106 m²/año a nivel regional. En este sentido, se ha estimado que el agua de lluvia circula de la siguiente manera: de $100 \%$ de la precipitación, 80 a $90 \%$ se infiltra y $10 \%$ se evapora en la superficie; el $70 \%$ de agua infiltrada se evapotranspira por las plantas y sólo aproximadamente $20 \%$ del agua de lluvia recarga el acuífero [Herrera y Comín, 2000: 204].

Sin embargo, esto no puede operar de forma homogénea en la PY, sobre todo después de analizar las evidencias obtenidas por Steinich et al. (1996: I58) y Steinich y Marín (1997:325) y conociendo que hay diferencias muy locales en cuanto a tipos y características de los sistemas. Es necesario puntualizar aquí (de acuerdo con la problemática presentada) que este balance probablemente pueda ser operativo desde un punto de vista regional, pero no local y además puede ser cuestionable. 


\section{¿Qué factores limitan elconocimiento del \\ balance hídrico de los cenotes a nivel local?}

El flujo subterráneo aún no esta bien definido y existen muchas dudas acerca a la circulación subterránea del agua de la PY. Existen al menos tres hipótesis que tratan de explicarlo:

a) Doehring y Butler (1974: 593) y la Comisión Nacional del Agua (CNA, 2002) sugieren un patrón de circulación radial, es decir desde el centro a la periferia de la PY.

b) Herrera y Comín (2000: 222) establecen un patrón diferente: una principal del centro hacia el norte, una secundaria sin un flujo claro y por último una al sur de Campeche hacia el Golfo de México.

c) Suárez y Rivera (2000: I57) marcan un flujo dominante desde el sur, hacia el este y norte de la misma.

Estas diferencias en opinión se deben a que los patrones de flujo se han construido exclusivamente por las diferencias en altitud de la PY.

Por lo tanto, esta información refleja el desconocimiento y confusión acerca del flujo subterráneo del agua a escala regional, incluso algunos autores han generalizado el comportamiento de la circulación subterránea del agua para toda la PY (Herrera y Comín, 2000: 222) siendo que existen zonas (parte Este y Oeste de la PY), con escaso o nulo trabajo a este respecto.

Ahora cabe preguntarse, ies posible obtener un balance hídrico detallado de los sistemas cársticos que sea funcional tanto a escala regional como local? Con la información recabada hasta hoy no parece una tarea tan sencilla, sin embargo, se pueden dar algunas sugerencias para determinarlo; por ejemplo se deben de identificar aquellos parámetros ambientales considerados como Elementos Traza (ET).

Los ET pueden agruparse en tres categorías: I) aquellos que tienen alta variabilidad espacial y temporal y pueden cambiar significativamente entre un sistema y otro; por ejemplo $\mathrm{NO}_{3}, \mathrm{Na}-, \mathrm{K}+, \mathrm{Mg}+, \mathrm{Cl}$ - y Sulfatos (reactivos); 2) aquellos con poca variación y una distribución homogénea espacial y temporalmente (no reactivos) por ejemplo el $\mathrm{pH}, \mathrm{Ca}++, \mathrm{HCO}_{3} ; \mathrm{y} 3$ ) los conservativos como el oxígeno isotópico (Socki et al., 2002: I8I2; Perrin et al., 2003: 677). Los ET han sido una herramienta importante para evaluar el flujo subterráneo, 
evapotranspiración y escorrentía en otras regiones cársticas (Perrin et al., op. cit.). De hecho, estos autores encontraron en 21 sistemas cársticos de Suiza, que el $\mathrm{pH}$ y el $\mathrm{Ca}++$, mostraron menor variación espacial y temporal.

Los elementos traza-reactivos están asociados a fuentes de contaminación, debido al uso excesivo de fertilizantes (Alcocer et al., 1998: 297). En sistemas cársticos de Suiza, se encontró que los sitios más contaminados fueron los que estaban asociados a tierras de cultivo (Perrin et al., 2003: 679). Así el K+, $\mathrm{Na}-, \mathrm{Mg}^{+}, \mathrm{SO}_{-4}, \mathrm{Cl}-$ y $\mathrm{NO}_{3}$, se asocian al uso de fertilizantes y su uso juega un papel importante en la variabilidad de la química del agua subterránea (Perrin et al., 2003). Estas observaciones son interesantes ya que se puede comenzar a mapear el flujo subterráneo de la PY a partir de las concentraciones de los elementos traza-reactivos. Por ejemplo, Herrera (1994: 47) encontró que la principal fuente de nitratos y silicatos de una laguna costera de Yucatán proviene de los manantiales de la zona.

\section{Conclusiones}

El conocimiento generado en las últimas tres décadas ha permitido proponer un balance hídrico para la PY; sin embargo, es cuestionable debido a que no se han considerado diferencias del flujo subterráneo, precipitación, capacidad de absorción del suelo según su edad geológica y grado de carstificación, velocidad de descarga, etc., a una escala regional y menos aún local. Es necesario incrementar los estudios hidrogeológicos, físicos, químicos y de contaminación en la PY, así como aprovechar la experiencia aplicada a otros sistemas cársticos de otras regiones. Es importante localizar los posibles tributarios, así como identificar y utilizar elementos-traza (reactivos, no-reactivos y conservativos), herramientas importantes para inferir sobre la residencia del agua y la geometría del flujo del sistema. El uso de fertilizantes como indicadores de contaminación y posible detección en los afloramientos superficiales (cenotes, lagunas) cercanos o lejanos, podrían ayudar a mapear los flujos subterráneos locales y posiblemente regionales, conforme se incremente la escala.

La disponibilidad del recurso agua aún no es problema para la región, (esto explica en parte el balance hídrico propuesto, así como las diferentes hipótesis en cuanto al flujo subterráneo de la PY), sin embargo, debido a la intrincada geohidrología de la región, queda una gran tarea por hacer en estas regiones cársticas. 
Alcocer, J., Lugo, A., Marín, L. y Escobar, E. (1998). "Hydrochemistry of waters from five cenotes and evaluation of their suitability for drinkingwatersupplies, northeastern Yucatán, Mexico”. Hydrgeology Journal, 6, 293-301.

Alcocer, J.y Escobar, E. (1996).“Limnological regionalization of Mexico”. Lakes and Reservoirs: Research and Management, 2, 55-69.

Armengol, D. (1997). “Caracterización estructural del zooplancton de las lagunas cársticas de cuenca con especial atención a su distribución vertical”. Tesis doctoral. España: Universidad de Valencia, 253 p.

Cervantes, A., Elías, M. y Suárez, E. (2002). “Limnological and morphometrical data of eight karstic systems 'cenotes' of the Yucatan Peninsula, Mexico, during the dry season (febrero-mayo, 200 I)". Hidrobiología, 482, 167-177.

Comisión Nacional del Agua. (2002). Región XII, Península de Yucatán. México: CNA, 16 p.

Doehring, D. y Butler, J. (1974). "Hydrogeologic constraints on Yucatan developmen”. Science, I86, 59|-595.

Herrera, J. (1994). “Correlaciones de parámetros hidrobiólogicos de la laguna de Celestún, Yucatán”. Anales del Instituto de Ciencias del Mar y Limnología, 2I, 43-53.

Herrera, J. y Comín, F. (2000). "An introductory account of types of aquatic ecosystems of Yucatan Peninsula (SE México)”, en Munawar M., S. Lawrence, I. y D. Malley (ed.). Aquatic ecosystems of Mexico. Status and scope. The Netherlands: Backhuys Publishers, 213-227.

Lavat, D., Ababou, R. y Mongin, A. (200I). "Introduction of wavelet analysis to rainfall/runoffs relationship for a karstic basin: The case of LieqAtherey karstic system (France)". Ground Water, 39, 605-6I7.

Lesser,J.yWeidie,A. (1988).“Region 25,Yucatan Peninsula”, en Back,W., Rosenshein, J. y Seaber, P. (ed.). Hydrogeology. Boulder, Colorado: The Geology of North America USA: Geologycal Society of America, O-2

Martin,J.y Screaton, E.(200I)."Exchange of matrix and conduit water with examples from the Florida aquifer”, en E. Kunianski (ed.). U.S. Geological survey Karst interest group proceedings, water resources investigation report 0I-40 I , 38-44. 
Maramathas,A., Maroulis, Z.y Marinos, D. (2003).“Brackish karstic springs model: Application to Almiros spring in Crete". Ground Water, 4I, 608-6I9. Medina, I. y Herrera, J. (2003). "Spatial characterization of water quality in a karstic coastal lagoon without anthropogenic disturbance: a multivariate approach". Estuarine Coastal and Shelf Science, 58, 455465.

Murat, H. (1998). "Water balance and water quality in the Curuksu basin, western Turkey". Hydrogeology Journal, 7, 405-4I8.

Perrin, J., Jeannin,Y.y Zwahlen, F. (2003).“Implications of the spatial variability of infiltration-water chemistry for the investigation of karstic aquifers: a field study at Milandre test site, Swiss Jura". Hydrogeology Journal, II, 673-686.

Schmitter,J., Comín, F., Escobar, E., Herrera,J.,Alcocer,J., Suárez, E., Elías, M., Díaz, V., Marín, L. y Steinich, B. (2002). "Hydrogeochemical and biological characteristics of cenotes in the Yucatan Peninsula (SE Mexico)". Hydrobiología, 467, 21 5-228.

Steinich B., Olimán, G., Marín, L.y Perry, E. (1996).“Determination of the ground water divide in the karst aquifer of Yucatan, Mexico, combining geochemical and hydrogeological data". Geofisica Internacional, 35, I53-159.

Steinich, B. y Marín, L. (1997). "Determination of flow characteristics in the aquifer of the Northwestern Peninsula of Yucatan, Mexico". Journal of Hydrobiology, 191, 315-331.

Socki, R. Perry, Jr. y Romanek, C. (2002). "Stable isotope systematics of two cenotes from the northern Yucatan Peninsula, Mexico". Limnology and Oceanography, 47, 1808-1818.

Suárez, E. y Rivera. (2000). "The aquatic fauna of karstic environments in the Yucatan Peninsula, Mexico: an updated overview”, en Munawar M., Lawrence, Malley, S.y D. (ed.). Aquatic ecosystems of Mexico. Status and scope. The Netherlands: Backhuys Publishers, I5 I-I64. 